\title{
Cocoa, Blood Pressure, and Vascular Function
}

\author{
Valeria Ludovici ${ }^{1,2}$, Jens Barthelmes' ${ }^{1}$, Matthias P. Nägele', Frank Enseleit', \\ Claudio Ferri' ${ }^{2}$, Andreas J. Flammer', Frank Ruschitzka ${ }^{1}$ and Isabella Sudano ${ }^{1 *}$ \\ ${ }^{1}$ Cardiology, University Heart Center, University Hospital and University of Zurich, Zurich, Switzerland, \\ ${ }^{2}$ Department of Life, Health and Environmental Sciences, University of L'Aquila, L'Aquila, Italy
}

OPEN ACCESS

Edited by:

Emilio Jirillo,

Università degli studi di

Bari Aldo Moro, Italy

Reviewed by:

Honglin Dong,

Coventry University,

United Kingdom

Nicolò Merendino,

Università degli Studi della

Tuscia, Italy

Siu-Lung Chan,

University of Vermont,

United States

*Correspondence:

Isabella Sudano

isabella.sudano@usz.ch

Specialty section:

This article was submitted to

Nutritional Immunology,

a section of the journal

Frontiers in Nutrition

Received: 06 April 2017

Accepted: 10 July 2017

Published: 02 August 2017

Citation:

Ludovici V, Barthelmes J, Nägele MP, Enseleit F, Ferri C, Flammer AJ,

Ruschitzka F and Sudano I (2017) Cocoa, Blood Pressure, and Vascular Function.

Front. Nutr. 4:36.

doi: 10.3389/fnut.2017.00036
Cardiovascular disease (CVD) represents the most common cause of death worldwide. The consumption of natural polyphenol-rich foods, and cocoa in particular, has been related to a reduced risk of CVD, including coronary heart disease and stroke. Intervention studies strongly suggest that cocoa exerts a beneficial impact on cardiovascular health, through the reduction of blood pressure (BP), improvement of vascular function, modulation of lipid and glucose metabolism, and reduction of platelet aggregation. These potentially beneficial effects have been shown in healthy subjects as well as in patients with risk factors (arterial hypertension, diabetes, and smoking) or established CVD (coronary heart disease or heart failure). Several potential mechanisms are supposed to be responsible for the positive effect of cocoa; among them activation of nitric oxide (NO) synthase, increased bioavailability of $\mathrm{NO}$ as well as antioxidant, and anti-inflammatory properties. It is the aim of this review to summarize the findings of cocoa and chocolate on BP and vascular function.

Keywords: cocoa, endothelial function, blood pressure, arterial stiffness, flavonoids

\section{INTRODUCTION}

Cardiovascular disease (CVD) represents the most common cause of death in the Western world, with an estimated 17.5 million people dying from CHD (coronary heart disease or stroke) every year (1). A nutraceutical approach has been proposed to counteract the increasing burden of CVD. The consumption of polyphenol-rich foods has been related to a lower risk of cardiovascular events (cardiovascular mortality, myocardial infarction, and stroke) both in the general population and in patients with cardiovascular risk factors in several interventional and epidemiological trials (2-5). Polyphenols are believed to be largely responsible for this protective role. Characterized as compounds with phenolic structural features (6), they are a class of natural bioactive substances with numerous anti-atherogenic properties including anti-inflammatory, anti-aggregating, and vasodilatory effects, the ability to lower blood pressure (BP), to prevent oxidation of low-density lipoprotein (LDL), and to improve glucose and lipid profiles (7).

Fruits, vegetables, tea, chocolate, and wine contain a high amount of polyphenols. Among them, cocoa beans are one of the richest known sources of flavonoids (8), and their protective properties have been recognized and used by several cultures among centuries. The origins of chocolate are usually traced back to the pre-Columbian populations, which were probably the first to cultivate cocoa plants. The consumption of cocoa, appreciated for its invigorating and healthy effects, differed from today: they used to dissolve dried cocoa beans in water, adding cinnamon and pepper to enhance their strong and bitter taste. With its arrival in Europe in the sixteenth century, cocoa was processed in 
a sweet soft beverage and rapidly became a luxury item. In the last century, the words cocoa and chocolate became intensely linked to hypertension, diabetes, overweight, and obesity (9). However, in the last two decades the salutary cardiovascular effects of this ancient medicinal food have begun to be reevaluated, and these properties have been related to cocoa's high content of flavonoids, members of the broader polyphenol class. The main constituents are flavanols, present as monomeric (-)-epicatechin and (+)-catechin, together with their dimers, oligomers, and polymers, the so-called proanthocyanidins, responsible for cocoa bitterness when complexing with salivary proteins (10). Although flavanols are likely to be responsible for cocoa's beneficial effects, they are lost during the conventional chocolate manufacturing process, so that the total flavanol content of commercial chocolate varies by more than 10-folds (11). Upon harvest, cocoa beans are usually fermented by environmental microbiota. This process creates flavor precursors that further develop during the roasting step, generating the peculiar cocoa flavors that define its quality (12). Fermentation and roasting significantly decreases the polyphenol and flavanol content of cocoa due to high temperature conditions and oxidation (13). Furthermore, alkalinization, used to modify cocoa color and give it a milder taste, results in a $60 \%$ decrease of total flavanol content (14). In humans, flavanol serum concentration increases in a dose-dependent manner after ingestion, reaching its peak usually $2-3 \mathrm{~h}$ after cocoa intake $(15,16)$. Flavanols are still detectable in plasma $8 \mathrm{~h}$ after consumption (17). Cocoa is also rich in theobromine, a 3,7-dimethylated xanthine alkaloid, and minerals such as potassium or magnesium (18).

Several epidemiological (19-21) and interventional studies strongly suggest that cocoa consumption, as well as vegetables and fruit intake, has numerous beneficial effects on cardiovascular health, including lowering of BP (22), improving vascular function (23), reducing of platelet aggregation and adhesion (24), having anti-inflammatory properties (25), and improving glucose and lipid metabolism (26). These positive effects have been found in healthy subjects (27) as well as in patients with risk factors (arterial hypertension, diabetes, and smoking) (28) or established CVD (coronary heart disease or heart failure) (29). Various potential mechanisms, including the increased bioavailability of nitric oxide (NO) and the anti-inflammatory and antioxidant effect, are supposed to be responsible for the protective properties of cocoa (30).

This review aims to summarize the effects of cocoa and chocolate on BP and vascular function.

\section{EPIDEMIOLOGICAL EVIDENCE}

After observing the Kuna Indians from Central America, researchers discovered how cocoa might be able to lower BP and extend life expectancy (31). Indeed, this population surprisingly had a very low incidence of hypertension when aging, despite having a high salt diet compared to other normotensive communities (32). Interestingly, this was not related to genetic factors, since the same population, migrated from the San Blas islands to Panama City for economic reasons, showed to have BP levels similar to other urban dwelling people (31). In addition, when compared to other American citizens, a marked reduction in cardiovascular mortality was noticed (33). To explain this difference, many environmental factors were investigated, such as differences in lifestyle or tobacco use, but ruled out as contributories. Finally, it was found that Island-Kuna, but not Mainland-Kuna, used to drink five cups of cocoa per day, which, moreover, was determined to be flavonoid-rich (approximate intake $900 \mathrm{mg}$ per day, i.e., the suggested highest intake worldwide) (34).

Since then, many epidemiological studies confirmed the assumption that cocoa could be responsible for these findings. Flavonoid-rich foods intake, and particularly chocolate consumption, were associated with a lower risk of death due to CVD in the Iowa Women's Health Study (19). Subsequently, in the Zutphen Elderly Study, a similar reduction in cardiovascular mortality after chocolate consumption was reported. Comparing the groups with higher and lower chocolate intake, a reduction of $3.7 \mathrm{mmHg}$ in systolic BP (SBP; 95\% CI, -7.1 to $-0.3 \mathrm{mmHg} ; p=0.03$ ) and a reduction of $2.1 \mathrm{mmHg}$ in diastolic BP (DBP; 95\% CI, -4.0 to $-0.2 \mathrm{mmHg} ; p=0.03$ ) were observed. Higher chocolate intake was associated to significant reduction in cardiovascular mortality (adjusted relative risk 0.50, 95\% CI, 0.32-0.78; $p=0.004$ ) and all-cause mortality (adjusted relative risk 0.53, 95\% CI, 0.39-0.72; $p<0.001$ ) (20). In the Stockholm Heart Epidemiology Program, chocolate intake related to a reduced cardiovascular mortality after acute myocardial infarction (21). Furthermore, this reduction appeared to be dose dependent. Compared with those never consuming chocolate, the subjects consuming chocolate less than once, up to once or up to twice per week showed progressively decreasing hazard ratios for cardiac mortality $[0.73$ (95\% CI, $0.41-1.31), 0.56$ (0.32-0.99), and 0.34 (0.17-0.70), respectively]. However, chocolate consumption was weakly associated with a lower rate of total mortality and non-fatal outcomes (21). The same findings emerged in two cohort studies of middle-aged Swedish women and men, in which daily moderate chocolate intake had an inverse association with chronic heart failure hospitalization and death $(35,36)$. Furthermore, in the National Heart, Lung, and Blood Institute Family Heart Study, chocolate intake correlated inversely with prevalent coronary heart disease in a general United States population (37). The most recent epidemiological data coming from the analysis of the European Prospective Investigation into Cancer Norfolk cohort support the prior findings (38). When compared to those who consumed no chocolate, subjects in the highest quintile of chocolate intake (15.6-98.8 g per day) demonstrated a significantly reduced rate of stroke (HR 0.77, 95\% CI, 0.62-0.96) and cardiovascular mortality (HR 0.75, 95\% CI, 0.62-0.92). Similar results emerged from a meta-analysis of 9 separate studies involving 157,809 participants (38). However, it is important to underline that none of these epidemiological studies focused on the amount of cocoa intake. Thus, it is not possible to make efficient comparison between the abovementioned studies.

\section{COCOA AND BP: INTERVENTIONAL STUDIES}

Arterial hypertension is a major modifiable risk factor for cardiovascular and cerebrovascular disease (39). Every $10 \mathrm{mmHg}$ 
reduction in SBP significantly reduces the risk of major cardiovascular events, $\mathrm{CHD}$, stroke, and heart failure, which leads to a significant $13 \%$ reduction in all-cause mortality (40). To date, several interventional studies have assessed the efficacy of cocoa in lowering BP both in healthy subjects and in patients with cardiovascular risk factors (Table 1).

Compared to cocoa butter chocolate, the regular consumption (14 days) of flavanol-rich milk chocolate (168 mg flavanols) was significantly linked with the reduction of SBP $(-6 \mathrm{mmHg})$ and DBP $(-5 \mathrm{mmHg})$, as well as with LDL cholesterol and oxidative stress markers in 28 healthy individuals (41). Similarly, shortterm administration (7 days) of flavanol-rich dark chocolate significantly reduced SBP $(p<0.05)$ and insulin resistance $(p<0.001)$ in 20 healthy subjects (42). In 101 normotensive subjects, randomized to receive dark chocolate bars $(397 \mathrm{mg}$ flavanols), cocoa beverage (357 mg flavanols), or matching placebo for 6 weeks, the flavanol-rich chocolate consumption reduced SBP by $3.58 \mathrm{mmHg}$, with no significant effect on DBP (43). Furthermore, in 90 "healthy" elderly subjects, a statistically significant improvement in BP $(p<0.0001)$, insulin resistance $(p<0.0001)$, and lipid peroxidation $(p=0.001)$ were seen after 8 weeks in the high flavanol (993 mg, SBP: $-7.83 \pm 0.56 \mathrm{mmHg}$, DBP: $-4.77 \pm 0.37 \mathrm{mmHg}$ ) and intermediate flavanol $(520 \mathrm{mg}$, SBP: $-6.8 \pm 0.59 \mathrm{mmHg}$, DBP: $-3.2 \pm 0.36 \mathrm{mmHg}$ ) intake groups in comparison to the low-flavanol group (48 mg, SBP: $-1.6 \pm 1.06 \mathrm{mmHg}$, DBP: $-1.57 \pm 0.61 \mathrm{mmHg})$. Moreover, flavanol consumption demonstrated a positive effect on cognitive performance (44). In the recently published Flaviola Health Study, 100 healthy subjects were enrolled and randomized to cocoa flavanol (CF) containing drink (450 mg) or CF-free drink for 1 month (27). CF intake decreased SBP and DBP by $4.4 \mathrm{mmHg}(95 \% \mathrm{CI}, 7.9-0.9 \mathrm{mmHg})$ and $3.9 \mathrm{mmHg}(95 \% \mathrm{CI}$, 6.7-0.9 $\mathrm{mmHg}$ ), improved endothelial function, and showed a positive effect on total and LDL cholesterol. By applying the

TABLE 1 | Studies investigating cocoa and blood pressure.

\begin{tabular}{|c|c|c|c|c|c|c|c|}
\hline Reference & Year & Study design & Population & $\begin{array}{l}\text { Duration } \\
\text { (weeks) }\end{array}$ & Intervention & $\begin{array}{c}\text { Reduction } \\
\text { of SBP/ } \\
\text { DBP active } \\
\text { group } \\
\text { (mmHg) }\end{array}$ & $\begin{array}{c}\text { Reduction } \\
\text { of SBP/DBP } \\
\text { control } \\
\text { group } \\
(\mathrm{mmHg})\end{array}$ \\
\hline Fraga et al. (41) & 2005 & Randomized crossover & 28 normotensive & 2 & High-flavanol milk (168 mg)/white chocolate & $-6 /-5$ & $-2 /-1$ \\
\hline Grassi et al. (42) & 2005 & Randomized crossover & 15 normotensive & 1 & Dark (500 mg flavanols)/white chocolate & $-7 /-4.2$ & $-0.5 /-0.3$ \\
\hline Crews et al. (43) & 2008 & $\begin{array}{l}\text { Randomized double-blind } \\
\text { parallel }\end{array}$ & 101 normotensive & 6 & $\begin{array}{l}\text { Dark chocolate (397 mg flavanol) and cocoa } \\
\text { drink ( } 357 \text { mg flavanol)/low-flavanol chocolate } \\
\text { and drink }\end{array}$ & $-3.58 /-0.5$ & $-3.05 /-0.57$ \\
\hline $\begin{array}{l}\text { Mastroiacovo } \\
\text { et al. (44) }\end{array}$ & 2015 & $\begin{array}{l}\text { Randomized double-blind } \\
\text { parallel }\end{array}$ & 90 normotensive & 8 & $\begin{array}{l}\text { High (993 mg)/intermediate (520 mg)/low } \\
\text { (48 mg) flavanol cocoa drink }\end{array}$ & $-7.83 /-4.77$ & $-1.60 /-1.57$ \\
\hline Sansone et al. (27) & 2015 & $\begin{array}{l}\text { Randomized double-blind } \\
\text { parallel }\end{array}$ & 100 normotensive & 4 & High (450 mg)/low-flavanol cocoa drink & $-4.4 /-3.9$ & $-1 / 0$ \\
\hline Grassi et al. (23) & 2015 & $\begin{array}{l}\text { Randomized double-blind } \\
\text { crossover }\end{array}$ & 20 normotensive & 1 & $\begin{array}{l}\text { High }(800,500,200,80 \text { mg)/low }(0 \text { mg) } \\
\text { flavanol cocoa }\end{array}$ & $-4.8 /-3.03$ & $-1-$ \\
\hline Murphy et al. (45) & 2003 & $\begin{array}{l}\text { Randomized double-blind } \\
\text { parallel }\end{array}$ & 32 normotensive & 4 & $\begin{array}{l}\text { High ( } 234 \text { mg flavanols and procyanidins)/low- } \\
\text { flavanol chocolate }\end{array}$ & $+2 /-1$ & $+3 / 0$ \\
\hline Engler et al. (46) & 2004 & Randomized double-blind & 21 normotensive & 2 & $\begin{array}{l}\text { High ( } 213 \text { mg procyanidins, } 46 \text { mg } \\
\text { epicatechin)/low-flavanol chocolate }\end{array}$ & $-1 /+0.9$ & $-2.8 /-0.1$ \\
\hline Shiina et al. (47) & 2009 & Randomized single-blind & 39 normotensive & 2 & Dark (550 mg flavanols)/white chocolate & $+4.6 /+6.6$ & $+4 /+5.2$ \\
\hline Njike et al. (48) & 2011 & Randomized crossover & $\begin{array}{l}44 \text { normotensive } \\
\text { overweight }\end{array}$ & 6 & High/low-flavanol cocoa drink & $+2.2 /-0.5$ & $-0.1 /+0.8$ \\
\hline Taubert et al. (49) & 2003 & Randomized crossover & 13 hypertensive & 2 & Dark (500 mg flavanols)/white chocolate & $-5.1 /-1.8$ & $+0.4 /+0.3$ \\
\hline Taubert et al. (50) & 2007 & $\begin{array}{l}\text { Randomized single-blind } \\
\text { parallel }\end{array}$ & $\begin{array}{l}44 \text { pre-hypertensive/ } \\
\text { hypertensive }\end{array}$ & 18 & Dark(30 mg flavanols)/white chocolate & $-2.9 /-1.9$ & $+0.1 / 0$ \\
\hline Grassi et al. (51) & 2005 & Randomized crossover & 20 hypertensive & 2 & Dark (500 mg flavanols)/white chocolate & $-11.9 /-8.5$ & $-0.7 /-0.6$ \\
\hline $\begin{array}{l}\text { Muniyappa et al. } \\
\text { (52) }\end{array}$ & 2008 & $\begin{array}{l}\text { Randomized double-blind } \\
\text { crossover }\end{array}$ & 20 hypertensive & 2 & High (900 mg)/low-flavanol drink & $-2 /-3$ & $-1 /-4$ \\
\hline Davison et al. (53) & 2010 & $\begin{array}{l}\text { Randomized double-blind } \\
\text { crossover }\end{array}$ & 52 hypertensive & 6 & High $(1,052 \mathrm{mg}) / \mathrm{low}$-flavanol cocoa drink & $-5.3 /-3$ & $-2.1 /+0.1$ \\
\hline Grassi et al. (54) & 2008 & Randomized crossover & $\begin{array}{l}19 \text { hypertensive } \\
\text { with IGT }\end{array}$ & 2 & Dark (1,008 mg polyphenols)/white chocolate & $-3.8 /-3.9$ & $-0.1 /-0.2$ \\
\hline Davison et al. (55) & 2008 & Randomized parallel & $\begin{array}{l}49 \text { normotensive } \\
\text { obese or overweight }\end{array}$ & 12 & High (902 mg)/low-flavanol cocoa & $-1.9 /-1.8$ & $+4.2 /+2.8$ \\
\hline Rostami et al. (28) & 2015 & Randomized double-blind & $\begin{array}{l}60 \text { hypertensive } \\
\text { diabetic }\end{array}$ & 8 & Dark/white chocolate & $-5.9 /-6.4$ & $-1.1 /+0.2$ \\
\hline $\begin{array}{l}\text { Monagas et al. } \\
(56)\end{array}$ & 2009 & Randomized crossover & $\begin{array}{l}47 \text { diabetics or } \\
\text { more than } 3 \mathrm{CV} \text { risk } \\
\text { factors }\end{array}$ & 4 & $\begin{array}{l}\text { Cocoa powder (495 mg polyphenols) with } \\
\text { milk/milk }\end{array}$ & $0.0 /-2$ & $-3 /-3$ \\
\hline $\begin{array}{l}\text { De Palma et al. } \\
\text { (57) }\end{array}$ & 2016 & Randomized crossover & $\begin{array}{l}32 \text { patients with } \\
\text { stable HF }\end{array}$ & 4 & High $(1,064 \mathrm{mg}) / \mathrm{low}$-flavanol dark chocolate & $-1.8 /-4.2$ & $-0.9 /+2.9$ \\
\hline
\end{tabular}

IGT, impaired glucose tolerance; HF, heart failure; CHD, coronary heart disease; CV, cardiovascular; SBP, systolic blood pressure; DBP, diastolic blood pressure. 
Framingham Risk Score, CF intake significantly lowered the 10 -year risk for CHD, CVD, and cardiovascular death in so-far healthy people (27). Moreover, Grassi and colleagues recently demonstrated that 1 week of supplementation with either 80 or $200 \mathrm{mg}$ total flavanols (17 or $42 \mathrm{mg}$ epicatechin, respectively) significantly decreased BP in healthy individuals (23). In particular, they documented a decrease in BP (SBP: $-4.8 \pm 1.03 \mathrm{mmHg}$, $p<0.0001$; DBP: $-3.03 \pm 1.07 \mathrm{mmHg}, p=0.0011$ ), and an improvement in endothelial function (23). However, in four different studies, flavanol-rich cocoa $(234,259,550,805 \mathrm{mg}$ flavanols per day, respectively) did not improve BP levels compared to placebo in normotensive subjects (45-48).

The effect of cocoa consumption on BP was also assessed in hypertensive patients. In a randomized crossover trial, 13 hypertensive patients were randomized to dark polyphenol-rich chocolate (500 mg polyphenols) or white chocolate. After 14 days, only polyphenol-rich chocolate decreased SBP by $5.1 \mathrm{mmHg}$ and DBP by $1.8 \mathrm{mmHg}$; after consumption discontinuation, BP levels returned to pre-intervention values within 2 days (49). Moreover, in 46 mildly hypertensive patients, low chronic cocoa intake (30 mg flavanols per day for 18 weeks) reduced SBP by $-2.9 \pm 1.6 \mathrm{mmHg}(p<0.001)$ and DBP by $-1.9 \pm 1.0 \mathrm{mmHg}$ $(p<0.001)$ compared to placebo (50). In a crossover study, 20 subjects with never treated essential hypertension were randomized to dark (500 mg flavanols) or white chocolate (flavanol free) for 15 days. Dark chocolate decreased ambulatory BP (24-h SBP: $-11.9 \pm 7.7 \mathrm{mmHg}, p<0.0001 ; 24-\mathrm{h} \mathrm{DBP}:-8.5 \pm 5.0 \mathrm{mmHg}$, $p<0.0001)(p<0.0001)$, serum LDL cholesterol $(p<0.05)$, and improved vascular function (51). However, in a different crossover study enrolling 20 hypertensive subjects, flavanol-rich cocoa intake ( $900 \mathrm{mg}$ per day) did not improve BP after 2 weeks, compared to placebo (52). A subsequent study evaluated the minimum dose required for BP lowering. A population of 52 subjects with untreated mild arterial hypertension was randomized to receive cocoa beverage containing different doses of flavanols $(33,372,712$, or 1,052 mg per day, respectively). After 6 weeks, only the highest flavanol dose (1,052 mg per day) demonstrated a significant reduction in $24-\mathrm{h} \mathrm{SBP}(5.3 \pm 5.1 \mathrm{mmHg}$; $p=0.001)$, DBP $(3 \pm 3.2 \mathrm{mmHg} ; p=0.002)$, and mean arterial $\mathrm{BP}(3.8 \pm 3.2 \mathrm{mmHg} ; p=0.0004)(53)$.

Flavanol intake also demonstrated a positive effect in hypertensive patients with impaired glucose tolerance (IGT) and diabetes mellitus. In particular, 19 hypertensive patients with IGT were randomized to receive flavanol-rich dark chocolate or flavanol-free white chocolate for 15 days. Dark chocolate reduced both SBP and DBP (SBP: $-3.82 \pm 2.40 \mathrm{mmHg}$; 24-h SBP: $-4.52 \pm 3.94$ mmHg; DBP: $-3.92 \pm 1.98$ mmHg; 24 -h DBP: $-4.17 \pm 3.29 \mathrm{mmHg})$, total and LDL cholesterol $(p<0.0001)$ as well as improved vascular function and insulin sensitivity $(p<0.05)$ (54). High-flavanol cocoa (902 mg) reduced SBP and DBP (SBP: $-1.9 \mathrm{mmHg}$, DBP: $-1.8 \mathrm{mmHg}, p<0.05$ ) and improved vascular function among overweight and obese adults (55). A recent study conducted on 60 subjects affected by hypertension and type 2 diabetes, randomized to flavanol-rich dark chocolate or white chocolate for 8 weeks, confirmed that flavanol-rich chocolate is effective in decreasing BP, fasting blood sugar, and triglyceride levels in patients with cardiovascular risk factors (28). However, in a high-risk population (three or more cardiovascular risk factors), flavanol-rich cocoa intake did not reduce BP values after 4 weeks (56).

Furthermore, in 24 heart failure patients, a 4 weeks consumption of high-flavanol dark chocolate (1,064 mg flavanols per day) significantly reduced DBP and NT-proBNP levels, compared to low-flavanol dark chocolate intake (57).

Several meta-analyses evaluated the available evidence on this topic. Ried and colleagues analyzed 13 studies, revealing a $\mathrm{BP}$ reduction after cocoa consumption (mean $\mathrm{BP}$ change $\pm \mathrm{SE}$ : SBP: $-3.2 \pm 1.9 \mathrm{mmHg}, p=0.001$; DBP: $-2.0 \pm 1.3 \mathrm{mmHg}$, $p=0.003)$. The effect maintained statistical significance only for the studies evaluating hypertensiveand pre-hypertensive patients (SBP: $-5.0 \pm 3.0 \mathrm{mmHg} ; p=0.0009$; DBP: $-2.7 \pm 2.2 \mathrm{mmHg}$, $p=0.01)(58)$. Desch and coworkers also provided a metaanalysis including 10 randomized controlled trials involving either healthy subjects or patients with prehypertension/stage 1 hypertension (297 individuals). Cocoa consumption was associated with a $4.5 \mathrm{mmHg}$ reduction $(95 \% \mathrm{CI},-5.9$ to -3.2 , $p<0.001)$ for SBP and a $2.5 \mathrm{mmHg}$ reduction $(95 \% \mathrm{CI},-3.9$ to $-1.2, p<0.001$ ) for DBP (59). In the same year, a Cochrane review showed that flavanol-rich chocolate and cocoa products may have a statistically significant effect in lowering BP by 2-3 $\mathrm{mmHg}$ in the short term [SBP $(95 \% \mathrm{CI}):-2.77 \mathrm{mmHg}$ $(-4.72$ to -0.82$), p=0.005 ; \mathrm{DBP}(95 \% \mathrm{CI}):-2.20 \mathrm{mmHg}$ $(-3.46$ to -0.93$), p=0.006$ ] (22). A recent published Cochrane review confirmed these findings, observing a small $(2 \mathrm{mmHg})$ decrease in BP in the short term, more pronounced in the prehypertensive/hypertensive population (60).

\section{COCOA AND VASCULAR FUNCTION: INTERVENTIONAL STUDIES}

Endothelium, the smooth, continuous inner lining of blood vessels, exhibits not only a barrier function but also synthetizes and releases a variety of vasoactive substances. Thus, the imbalance between vasodilating and vasoconstricting mediators results in endothelial dysfunction (61). The impairment of endothelial function is an early event in the development of atherosclerosis and is associated with CVD (62). Endothelial dysfunction of the forearm, as assessed by the flow-mediated dilation of the brachial artery (FMD), is recognized to be a powerful surrogate marker for cardiovascular events and cardiovascular mortality, both in healthy subjects (63) and in patients with CV risk factors (64). Thus, FMD has been used to evaluate the effects of different interventions on endothelial function. To date, various studies have subsequently assessed the effects of cocoa on vascular function (Table 2).

In 15 healthy subjects, CFs intake (1.4-10.9 mg/kg body weight) acutely improved FMD at 1, 2, 3, and $4 \mathrm{~h}$ after consumption. The improvement in vascular function was comparable to the one induced by nitrate intake (65). Flavanol-rich chocolate (213 mg procyanidins, $46 \mathrm{mg}$ epicatechin) consumption significantly improved FMD in 21 healthy subjects also over a 2-week period $(p=0.024)$. Moreover, plasma epicatechin concentrations markedly increased after 2 weeks in the active treatment group, 
TABLE 2 | Studies investigating cocoa and vascular function.

\begin{tabular}{|c|c|c|c|c|c|c|}
\hline Reference & Year & Study design & Population & Duration & Intervention & Outcome \\
\hline $\begin{array}{l}\text { Rodriguez- } \\
\text { Mateos et al. } \\
(65)\end{array}$ & 2015 & $\begin{array}{l}\text { Randomized double- } \\
\text { blind crossover }\end{array}$ & $\begin{array}{l}15 \text { healthy } \\
\text { subjects }\end{array}$ & $1,2,3,4 \mathrm{~h}$ & $\begin{array}{l}\text { CF-rich drink (1.4-10.9 mg/kg body weight) } \\
\text { vs. nitrate or nutrient-matched flavanol-free } \\
\text { drink }\end{array}$ & $\begin{array}{l}\text { Improvement in FMD after flavanol } \\
\text { and nitrate intake }\end{array}$ \\
\hline $\begin{array}{l}\text { Engler et al. } \\
(46)\end{array}$ & 2004 & $\begin{array}{l}\text { Randomized } \\
\text { double-blind } \\
\text { placebo-controlled }\end{array}$ & $\begin{array}{l}21 \text { heathy } \\
\text { subjects }\end{array}$ & 2 weeks & $\begin{array}{l}\text { High flavonoid chocolate ( } 213 \mathrm{mg} \\
\text { procyanidins, } 46 \mathrm{mg} \text { epicatechin) vs. low- } \\
\text { flavonoid chocolate }\end{array}$ & $\begin{array}{l}\text { Improvement in FMD, increased } \\
\text { epicatechin concentrations }\end{array}$ \\
\hline $\begin{array}{l}\text { Sansone et al. } \\
(27)\end{array}$ & 2015 & $\begin{array}{l}\text { Randomized double- } \\
\text { blind controlled } \\
\text { parallel }\end{array}$ & $\begin{array}{l}100 \text { healthy } \\
\text { subjects }\end{array}$ & 30 days & $\begin{array}{l}\text { CF-containing drink ( } 450 \text { mg) or a nutrient- } \\
\text { matched flavanol-free control bi-daily }\end{array}$ & $\begin{array}{l}\text { Improvement in FMD, decreased SBP } \\
\text { and DBP, decreased PWV }\end{array}$ \\
\hline $\begin{array}{l}\text { Grassi et al. } \\
\text { (23) }\end{array}$ & 2015 & $\begin{array}{l}\text { Randomized double- } \\
\text { blind crossover } \\
\text { controlled }\end{array}$ & $\begin{array}{l}20 \text { healthy } \\
\text { subjects }\end{array}$ & 5 weeks & $\begin{array}{l}\text { Five treatments with daily intake of } 10 \mathrm{~g} \\
\text { cocoa }(0,80,200,500,800 \mathrm{mg} \text { flavonoids) }\end{array}$ & $\begin{array}{l}\text { Dose-dependent improvement in FMD, } \\
\text { decreased PWV, and BP }\end{array}$ \\
\hline $\begin{array}{l}\text { Schroeter } \\
\text { et al. (66) }\end{array}$ & 2006 & $\begin{array}{l}\text { Randomized } \\
\text { crossover }\end{array}$ & $\begin{array}{l}16 \text { healthy } \\
\text { subjects, } \\
\text { isolated rabbit } \\
\text { rings }\end{array}$ & $2 \mathrm{~h}$ & Drink with high flavonoid content & $\begin{array}{l}\text { Improvement in FMD, paralleled the } \\
\text { appearance of flavanols in plasma }\end{array}$ \\
\hline $\begin{array}{l}\text { Heiss et al. } \\
(67)\end{array}$ & 2015 & $\begin{array}{l}\text { Randomized double- } \\
\text { blind controlled } \\
\text { parallel }\end{array}$ & $\begin{array}{l}42 \text { healthy } \\
\text { subjects }\end{array}$ & 14 days & $\begin{array}{l}\text { CF-containing drink ( } 450 \mathrm{mg} \text { bid) vs. CF-free } \\
\text { drink }\end{array}$ & $\begin{array}{l}\text { Improvement in FMD, decreased PWV, } \\
\text { and in total peripheral resistances }\end{array}$ \\
\hline $\begin{array}{l}\text { Shiina et al. } \\
(47)\end{array}$ & 2009 & $\begin{array}{l}\text { Randomized } \\
\text { single-blind }\end{array}$ & $\begin{array}{l}39 \text { healthy } \\
\text { subjects }\end{array}$ & 2 weeks & $\begin{array}{l}45 \mathrm{~g} \text { commercially available dark chocolate } \\
\text { vs. white chocolate }\end{array}$ & $\begin{array}{l}\text { Improvement in coronary circulation as } \\
\text { measured by coronary velocity flow reserve }\end{array}$ \\
\hline $\begin{array}{l}\text { Grassi et al. } \\
(51)\end{array}$ & 2005 & $\begin{array}{l}\text { Randomized } \\
\text { crossover } \\
\text { placebo-controlled }\end{array}$ & $\begin{array}{l}20 \text { untreated } \\
\text { hypertensive } \\
\text { patients }\end{array}$ & 15 days & $\begin{array}{l}100 \mathrm{~g} \text { dark chocolate ( } 21.91 \mathrm{mg} \text { catechin, } \\
65,97 \mathrm{mg} \text { epicatechin) vs. flavanol-free white } \\
\text { chocolate }\end{array}$ & $\begin{array}{l}\text { Improvement in FMD, decreased BP and } \\
\text { LDL cholesterol, increased insulin sensitivity }\end{array}$ \\
\hline $\begin{array}{l}\text { Grassi et al. } \\
(54)\end{array}$ & 2008 & $\begin{array}{l}\text { Randomized } \\
\text { crossover } \\
\text { placebo-controlled }\end{array}$ & $\begin{array}{l}19 \\
\text { hypertensive } \\
\text { with IGT }\end{array}$ & 15 days & $\begin{array}{l}100 \mathrm{~g} \text { dark chocolate ( } 36 \mathrm{mg} \text { catechin, } \\
110 \text { mg epicatechin) vs. flavanol-free white } \\
\text { chocolate }\end{array}$ & $\begin{array}{l}\text { Improvement in FMD, decreased SBP } \\
\text { and DBP, decreased insulin resistance }\end{array}$ \\
\hline $\begin{array}{l}\text { Heiss et al. } \\
\text { (69) }\end{array}$ & 2005 & $\begin{array}{l}\text { Randomized double- } \\
\text { blind crossover }\end{array}$ & 11 smokers & $2 \mathrm{~h}$ & $\begin{array}{l}100 \mathrm{ml} \text { cocoa drink with high (176-185 mg) } \\
\text { or low (<11 mg) flavanol content }\end{array}$ & $\begin{array}{l}\text { Improvement in FMD and increased } \\
\text { circulating NO pool. Increased flavanol } \\
\text { metabolites }\end{array}$ \\
\hline $\begin{array}{l}\text { Hermann et al. } \\
\text { (70) }\end{array}$ & 2006 & $\begin{array}{l}\text { Randomized } \\
\text { placebo-controlled }\end{array}$ & 20 smokers & $2 \mathrm{~h}$ & $\begin{array}{l}40 \mathrm{~g} \text { commercially available dark chocolate } \\
\text { vs. white chocolate }\end{array}$ & $\begin{array}{l}\text { Improvement in FMD, antioxidant status, } \\
\text { and platelet function }\end{array}$ \\
\hline $\begin{array}{l}\text { Davison et al. } \\
\text { (55) }\end{array}$ & 2008 & $\begin{array}{l}\text { Randomized double- } \\
\text { blind placebo- } \\
\text { controlled parallel }\end{array}$ & $\begin{array}{l}49 \text { obese and } \\
\text { overweight } \\
\text { patients }\end{array}$ & 12 weeks & $\begin{array}{l}\text { Dietary high (902 mg) vs. low (36 mg) } \\
\text { flavanol intake }\end{array}$ & Improvement in FMD \\
\hline Njike et al. (48) & 2011 & $\begin{array}{l}\text { Randomized } \\
\text { controlled crossover }\end{array}$ & $\begin{array}{l}44 \text { overweight } \\
\text { patients }\end{array}$ & 6 weeks & $\begin{array}{l}\text { Sugar-free cocoa beverage or placebo, } \\
\text { sugar-sweetened cocoa beverage or } \\
\text { placebo }\end{array}$ & $\begin{array}{l}\text { Improvement in FMD, no change } \\
\text { in weight }\end{array}$ \\
\hline $\begin{array}{l}\text { West et al. } \\
\text { (71) }\end{array}$ & 2014 & $\begin{array}{l}\text { Randomized double- } \\
\text { blind crossover } \\
\text { placebo-controlled }\end{array}$ & $\begin{array}{l}30 \text { overweight } \\
\text { patients }\end{array}$ & 30 days & $\begin{array}{l}37 \mathrm{~g} \text { dark chocolate plus sugar-free cocoa } \\
\text { beverage (flavanols } 814 \mathrm{mg} \text { ) vs. low-flavanol } \\
\text { chocolate bar and cocoa-free and sugar- } \\
\text { free beverage }\end{array}$ & $\begin{array}{l}\text { Unchanged FMD, increased basal diameter } \\
\text { and peak diameter of the brachial artery, } \\
\text { increased basal blood flow, in women } \\
\text { decreased augmentation index }\end{array}$ \\
\hline $\begin{array}{l}\text { Balzer et al. } \\
(72)\end{array}$ & 2008 & $\begin{array}{l}\text { Randomized } \\
\text { double-blind }\end{array}$ & $\begin{array}{l}41 \text { diabetic } \\
\text { patients }\end{array}$ & 30 days & $\begin{array}{l}\text { Flavanol-rich cocoa }(321 \mathrm{mg} \text { flavanols } \times 3 \text { ) } \\
\text { or a nutrient-matched control }(25 \mathrm{mg} \\
\text { flavanols } \times 3 \text { ) }\end{array}$ & Improvement in FMD \\
\hline $\begin{array}{l}\text { Mellor et al. } \\
(73)\end{array}$ & 2013 & $\begin{array}{l}\text { Randomized double- } \\
\text { blind crossover } \\
\text { controlled }\end{array}$ & $\begin{array}{l}10 \text { diabetic } \\
\text { patients }\end{array}$ & $2 \mathrm{~h}$ & $\begin{array}{l}13.5 \mathrm{~g} \text { of high vs. low-flavanol chocolate; } \\
60 \text { min later, a } 75 \mathrm{~g} \text { oral glucose load }\end{array}$ & $\begin{array}{l}\text { Improved endothelial function assessed } \\
\text { by reactive hyperemia peripheral artery } \\
\text { tonometry }\end{array}$ \\
\hline $\begin{array}{l}\text { Heiss et al. } \\
(74)\end{array}$ & 2003 & $\begin{array}{l}\text { Randomized double- } \\
\text { blind crossover }\end{array}$ & $\begin{array}{l}20 \text { patients } \\
\text { with at least } 1 \\
\text { CV risk factor }\end{array}$ & $2 \mathrm{~h}$ & Flavanol-rich cocoa drink (100 ml) & $\begin{array}{l}\text { Improvement in FMD and increased levels } \\
\text { of nitrosated and nitrosylated species }\end{array}$ \\
\hline $\begin{array}{l}\text { Heiss et al. } \\
\text { (75) }\end{array}$ & 2010 & $\begin{array}{l}\text { Randomized double- } \\
\text { blind crossover } \\
\text { controlled }\end{array}$ & $\begin{array}{l}16 \mathrm{CHD} \\
\text { patients }\end{array}$ & 30 days & $\begin{array}{l}\text { Dietary high ( } 375 \text { mg bid) vs. low ( } 9 \text { mg bid) } \\
\text { flavanol cocoa drink }\end{array}$ & $\begin{array}{l}\text { Improvement in FMD and mobilization } \\
\text { of endothelial progenitor cells }\end{array}$ \\
\hline $\begin{array}{l}\text { Flammer et al. } \\
\text { (29) }\end{array}$ & 2012 & $\begin{array}{l}\text { Randomized } \\
\text { double-blind } \\
\text { placebo-controlled }\end{array}$ & $\begin{array}{l}20 \text { heart failure } \\
\text { patients }\end{array}$ & $\begin{array}{l}2 \mathrm{~h} \text { and } \\
30 \text { days }\end{array}$ & $\begin{array}{l}40 \mathrm{~g} \text { commercially available dark chocolate } \\
\text { vs. flavonoid-free placebo chocolate }\end{array}$ & Improvement in FMD of platelet function \\
\hline
\end{tabular}


TABLE 2 | Continued

\begin{tabular}{|c|c|c|c|c|c|c|}
\hline Reference & Year & Study design & Population & Duration & Intervention & Outcome \\
\hline $\begin{array}{l}\text { Flammer et al. } \\
\text { (76) }\end{array}$ & 2007 & $\begin{array}{l}\text { Randomized } \\
\text { double-blind }\end{array}$ & $\begin{array}{l}22 \text { heart } \\
\text { transplant } \\
\text { patients }\end{array}$ & $2 \mathrm{~h}$ & $\begin{array}{l}40 \mathrm{~g} \text { commercially available } \\
\text { dark chocolate vs. flavonoid-free } \\
\text { placebo chocolate }\end{array}$ & $\begin{array}{l}\text { Inducing coronary vasodilation, } \\
\text { improvement in coronary endothelial } \\
\text { function, and improvement } \\
\text { in platelet function }\end{array}$ \\
\hline $\begin{array}{l}\text { Rassaf et al. } \\
(77)\end{array}$ & 2016 & $\begin{array}{l}\text { Randomized } \\
\text { double-blind } \\
\text { placebo-controlled }\end{array}$ & $\begin{array}{l}57 \\
\text { hemodialytic } \\
\text { patients }\end{array}$ & 30 days & $\begin{array}{l}\text { CF-rich beverages ( } 900 \text { mg per } \\
\text { study day) vs. flavanol-free } \\
\text { beverages }\end{array}$ & $\begin{array}{l}\text { Improvement in FMD decreased DBP. } \\
\text { Ingestion of flavanols during HD alleviated } \\
\text { HD-induced vascular dysfunction }\end{array}$ \\
\hline $\begin{array}{l}\text { Sansone et al. } \\
\text { (68) }\end{array}$ & 2017 & $\begin{array}{l}\text { Randomized double- } \\
\text { blind crossover }\end{array}$ & $\begin{array}{l}47 \text { healthy } \\
\text { subjects }\end{array}$ & & $\begin{array}{l}\text { High }(820 \mathrm{mg}) / \mathrm{low} \text {-flavanol cocoa } \\
\text { drink with high }(220 \mathrm{mg}) / \mathrm{low} \\
\text { methylxanthines content }\end{array}$ & $\begin{array}{l}\text { CFs with methylxanthines increased } \\
\text { epicatechin serum concentration, } \\
\text { increased FMD decreased PWV } \\
\text { and DBP compared with flavanols alone }\end{array}$ \\
\hline
\end{tabular}

Modified from Ref. (116).

FMD, flow-mediated dilation; endoPAT, reactive hyperemia peripheral artery tonometry; PWV, pulse wave velocity; CV, cardiovascular; NO, nitric oxide; CHD, coronary heart disease; HD, hemodialysis; BP, blood pressure; SBP, systolic blood pressure; DBP, diastolic blood pressure; LDL, low-density lipoprotein; CF, cocoa flavanol; IGT, impaired glucose tolerance.

suggesting that the effect on vascular function was flavanolmediated (46). In the Flaviola Health Study, a 1-month CF intake increased FMD over control by $1.2 \%$ (95\% CI, 1.0-1.4) and decreased pulse wave velocity by $0.4 \mathrm{~m} / \mathrm{s}$ ( $95 \%$ CI, $0.8-0.04 \mathrm{~m} / \mathrm{s}$ ) (27). Furthermore, 20 healthy subjects were randomized to receive 5 treatments with daily intake of $10 \mathrm{~g}$ cocoa $(0,80,200$, 500 , and $800 \mathrm{mg}$ cocoa flavonoids per day) in 5 periods lasting 1 week. A dose-dependent increase in FMD (from 6.2\% to 7.3, $7.6,8.1,8.2 \%$ after the different flavonoid doses, respectively) was found (23). In healthy individuals, an improvement in vascular function after high-flavanol cocoa intake has been demonstrated in subsequent studies $(66,67)$. Flavanol-rich chocolate intake was also demonstrated to significantly improve coronary circulation in healthy adults, as assessed by coronary flow velocity reserve measurement with non-invasive transthoracic Doppler echocardiography (47). Interestingly, in a recently published randomized double-blind trial, an interaction between flavanols and methylxanthines, such as theobromine and caffeine contained in cocoa, has been identified. In particular, methylxanthines were demonstrated to increase epicatechin metabolites plasma levels, thus affecting flavanols absorption, and to enhance the positive vascular effect of flavanols (68).

Flavanol intake improved FMD in hypertensive patients with normal (51) as well as IGT (54). Flavanol-rich cocoa beverage also acutely improved endothelium-dependent vasodilation, platelet function, and circulating bioactive NO in smokers $(69,70)$. In 20 male smokers, dark chocolate, but not white chocolate, improved FMD after $2 \mathrm{~h}$ ( 7.0 vs. $4.4 \%, p=0.026$ ), and the effect lasted about $8 \mathrm{~h}$ after ingestion (70). Furthermore, a statistically significant reduction in $\mathrm{DBP}$ and mean $\mathrm{BP}$, and a parallel improvement in FMD was shown after high-flavanol cocoa intake in obese and overweight subjects (55). Moreover, the positive effect of cocoa consumption on endothelial function was not associated with weight gain (48). Conversely, in a subsequent study, an increase in basal and peak brachial artery diameter, with no consequent change in FMD, was assessed in a group of 30 overweight patients after 30 days high-flavanol chocolate intake (71). In a diabetic population, high-flavanol cocoa consumption was associated with statistically significant improvement in vascular function both acutely (after $2 \mathrm{~h}$ ) and chronically (30 days) in two different studies $(72,73)$. Flavanol-rich cocoa intake improved FMD (from 3.4 to $6.3 \%, p<0.001$ ) after 2 days in a population with at least one cardiovascular risk factor, including history of $\mathrm{CHD}$, hypertension, hyperlipidemia, diabetes, or current tobacco use (74). Altogether, these studies highlight the ability of CFs in improving vascular function in patients with cardiovascular risk factors.

The efficacy of flavanol intake on vascular function has been assessed also in a population of $16 \mathrm{CHD}$ patients, randomized to receive flavanol-rich or low-flavanol cocoa for 30 days. Results showed a significant improvement in endothelial function by $48 \%$, a decrease in SBP (mean change in active group: $-4.2 \pm 2.7 \mathrm{mmHg}$ ), and increasing levels of circulating angiogenic cells in the active treatment group compared with controls (75).

In a study from our group, 20 chronic heart failure patients were randomized to receive commercially available flavanol-rich chocolate or control chocolate (29). Flavanol-rich chocolate significantly improved FMD from baseline levels of $4.98 \pm 1.95$, to $5.98 \pm 2.32 \%$ ( $p=0.045$ and 0.02 for between-group changes) $2 \mathrm{~h}$ after intake, and to $6.86 \pm 1.76 \%$ after 4 weeks of daily consumption ( $p=0.03$ and 0.004 for between groups). After flavanol-rich chocolate intake, platelet adhesion significantly decreased in the short term, but the effect was not sustained after 4 weeks. BP values and heart rate did not change. We also assessed the effect of flavanol-rich dark chocolate compared with control chocolate on coronary vascular function in 22 heart transplant recipients, patients characterized by severely impaired vascular function (76). Two hours after ingestion, flavanol-rich dark chocolate but not control chocolate induced significant coronary vasodilation $(p<0.01)$, improved coronary vascular function $(p=0.01)$, and decreased platelet adhesion.

Cocoa flavanols demonstrated to have a protective role also among patients with end-stage renal disease (ESRD). A population of 57 ESRD patients was randomized to receive either flavanol-rich beverage $(900 \mathrm{mg})$ or placebo. Flavanol-rich cocoa improved endothelial function by 53\% $(p<0.001)$ and alleviated hemodialysis induced endothelial dysfunction $(p<0.001)$ after acute ingestion, with no effect on BP and heart rate. After 4 weeks of treatment, cocoa improved vascular function by $18 \%$ and decreased DBP $(p=0.03)(77)$. 
In a meta-analysis of 11 chronic and 11 acute studies, Hooper and colleagues found strong beneficial effects of cocoa on FMD, as well as reductions in DBP and mean arterial pressure. Chocolate or cocoa improved FMD regardless of the dose consumed (78).

Based on the epidemiological evidence and the results from interventional studies, the European Food Safety Authority (EFSA) published a health claim about the effect of polyphenols derived from cocoa on cardiovascular risk factors, assessing that CFs "help maintaining the elasticity of blood vessels, which contributes to normal blood flow." In order to obtain the claimed effect, they suggested "to consume $200 \mathrm{mg}$ of cocoa flavanols per day, provided by $2.5 \mathrm{~g}$ of high-flavanol cocoa powder or $10 \mathrm{~g}$ of high-flavanol dark chocolate, in the context of a balanced diet" (79). Conversely, Vlachojannis and coworkers asserted that only cocoa products with $100 \mathrm{mg}$ epicatechin or CF doses of around $900 \mathrm{mg}$ were able to achieve positive effects on FMD and BP, questioning the $200 \mathrm{mg}$ flavanols/46 mg epicatechin dose recommended by the EFSA (80).

Currently, the COcoa Supplement and Multivitamin Outcomes Study (COSMOS; NCT02422745) is ongoing to assess the capability of a cocoa extract supplement $(600 \mathrm{mg}$ per day flavanols/80 mg epicatechin) compared to a standard multivitamin supplement, to reduce the risk of CVD and cancer among men aged 60 years and older and women aged 65 years and older. Concomitantly, an ancillary study (COSMOS-Mind; NCT03035201) is being conducted to evaluate the effects of such supplements on cognitive function.

\section{PUTATIVE MECHANISMS}

The protective role of CFs intake on BP and endothelial function is likely to come from its vasodilatory effect; the underlying mechanisms are multiple and not fully understood (30).

In this context, the increased NO availability and the subsequent vasodilation may play a central role. In young spontaneously hypertensive rats, epicatechin delayed the occurrence of arterial hypertension and reduced locomotor hyperactivity; these results were both mediated by increased NO bioavailability and erythrocyte deformability (81). Taubert and colleagues (50) demonstrated that prolonged intake of small amounts of dark chocolate (6.3 g per day for 18 weeks) reduced BP and improved NO production in a population of 44 pre-hypertensive individuals. In particular, dark chocolate intake reduced mean SBP by $2.9 \mathrm{mmHg}$ $(p<0.001)$ and DBP by $1.9 \mathrm{mmHg}(p<0.001)$; these results were accompanied by a sustained increase of S-nitrosoglutathione, a source of bioavailable NO, by $0.23 \mathrm{nmol} / \mathrm{L}(p<0.001)(50)$. Flavanols, and particularly flavanol-rich cocoa, elevate NO bioavailability by both stimulating the NO synthase (eNOS) activity $(82,83)$ and increasing the availability of L-arginine (via reduction of its degradation by arginase) (84). Furthermore, in a rat model, flavanols prevented the elevation of BP induced by L-nitroarginine methyl ester (L-NAME), a powerful inhibitor of NOS (83). In addition, cocoa showed a similar inhibitor effect on endothelin-1 production, a powerful vasoconstrictor $(85,86)$. Flavanols proved capable to induce both NO-mediated and endothelium-derived hyperpolarizing factor-mediated relaxation in a large number of arteries including the coronary arteries (87). Since NO degradation is mediated by free radicals, the improvement in vascular function is also related to the anti-inflammatory and antioxidant properties of cocoa (88). In a systematic review of the literature on polyphenols and oxidative stress, four studies reported statistically significant improvements in markers of oxidative stress and inflammation after flavanol-rich cocoa intake (89). A significant reduction in oxidative stress occurred when dark chocolate was administered to smokers as opposed to milk chocolate (90). Furthermore, a high dose (472.5 mg) of flavonoids through cocoa powder led to reduction in all oxidative and inflammatory markers in type 2 diabetics, and to a parallel improvement in endothelial function $(1.7 \pm 0.1$ vs. $2.3 \pm 0.1 \%, p=0.01)$ assessed by reactive hyperemia peripheral artery tonometry (EndoPAT-2000) (73). After consumption of cocoa products, a decrease in markers of peroxidation was also observed in healthy subjects, as well as in obese, dyslipidemic, pre-hypertensive, and hypertensive patients (25). CFs directly scavenge ROS and nitrogen species (91); moreover, they modulate crucial enzymes related to oxidative stress, such as catalase, superoxide dismutase, glutathione peroxidase, glutathione reductase, glutathione transferase, xanthine oxidase, and lipooxygenase $(92,93)$. In line with this, the exposure of human endothelial cells to epicatechin inhibited endothelial NADPH oxidase, reduced superoxide and peroxynitrite levels, and consequently induced an increase in NO and cyclic guanosine monophosphate cellular levels (94). Furthermore, cocoa powder and epicatechin demonstrated to significantly decrease aortic oxidative stress and circulating markers related to impaired coagulation (von Willebrand factor, factor VIII, and fibrinogen) and inflammation (tissue necrosis factor- $\alpha$, interleukin-6, interleukin-10, and C-reactive protein) (95). In vitro experiments show that CFs inhibit pro-inflammatory cytokines such as interleukin-2, interleukin- $1 \beta$, and tissue necrosis factor- $\alpha$, and positively modulate the expression of anti-inflammatory cytokines, such as interleukin-4 and transforming growth factor- $\beta$ (92, 96-98).

An inhibition of angiotensin-converting enzyme (ACE) by cocoa constituents has been postulated. In a rat model, flavanol-rich cocoa powder significantly reduced BP in spontaneously hypertensive rats but did not exert a similar effect in normotensive Wistar-Kyoto rats. Interestingly, the effect of flavanol-rich cocoa $(300 \mathrm{mg} / \mathrm{kg}$ ) clearly mimicked that caused by captopril (50 mg/kg) (99). In 2006, Actis-Goretta and colleagues documented an in vitro interaction between cocoa flavonoids and ACE. They demonstrated that procyanidin-rich chocolate significantly inhibited purified ACE activity, whereas the inhibitory activity correlated with both the phenolic content $(p<0.003)$ and the flavanol content $(p<0.001)$. When incubated in membrane suspensions from rat kidney, chocolate $[634 \mu \mathrm{M}(+)$-catechin equivalents] high in procyanidin inhibited ACE activity by $70 \%$ and such low in procyanidin [314 $\mu \mathrm{M}(+)$-catechin equivalents] only inhibited ACE by $45 \%(p<0.001)$. The inhibition of ACE in tissue membrane suspensions was also observed in rat testes and lungs (100). In a subsequent study, extract from powdered cocoa beans was demonstrated to dose-dependently inhibit in vitro ACE activity; it also showed a dose-dependent radicals scavenging ability (101). Persson and colleagues (102) demonstrated that the acute consumption of dark chocolate (75 g, $72 \%$ of cocoa) inhibited ACE activity in vitro-after incubation in human endothelial 


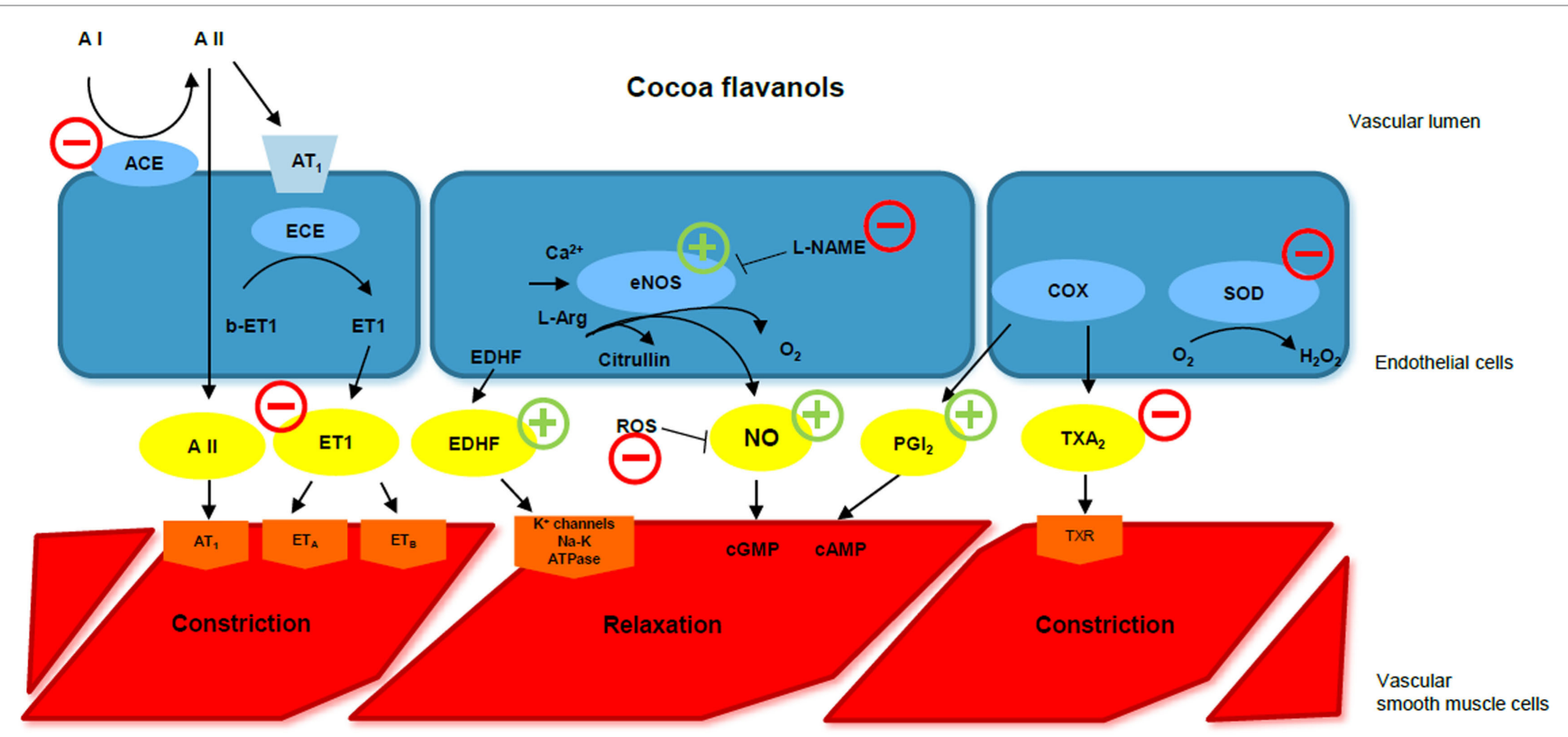

FIGURE 1 | Cocoa flavanols and endothelial function. ACE, angiotensin-converting enzyme; A I, angiotensin I; A II, angiotensin II; ECE, endothelin-converting enzyme; ET 1, endothelin-1; EDHF, endothelium-derived hyperpolarizing factor; eNOS, endothelial NO synthase; L-NAME, L-nitroarginine methyl ester; ROS, reactive oxygen species; NO, nitric oxide; cGMP, cyclic guanosine monophosphate; cAMP, cyclic adenosine monophosphate; COX, cyclooxygenase; PGI2, prostacyclin; TXA2, thromboxane A2; SOD, superoxide dismutase; CF, cocoa flavanol. CFs improve endothelial function via different pathways. They increase NO availability, stimulating eNOS function, preventing L-NAME-induced hypertension, and reducing ROS. They also stimulate EDHF-mediated relaxation, inhibit endothelin-1, and reduce ACE activity. Modified by Corti et al., Circulation 2009.

cells from umbilical veins (HUVEC) - and in vivo in 16 healthy volunteers. In HUVEC, a significant inhibition of ACE activity $(p<0.01)$ and an increase of NO levels $(p<0.001)$ were seen. In healthy subjects, dark chocolate significantly inhibited ACE activity (mean 18\%) $3 \mathrm{~h}$ after oral intake, with no relevant changes in circulating NO levels. According to ACE genotype, significant inhibition of ACE activity emerged in individuals with genotype insertion/insertion and deletion/deletion (mean 21 and $28 \%$, respectively) (102).

Recently, studies have begun to pay attention to the role of cocoa on mitochondria in cardiovascular health, as impaired mitochondrial function represents an early sign of endothelial dysfunction (103). The stimulation of mitochondrial function and biogenesis could ameliorate bioenergetic and metabolic status of cells, thereby improving vascular function and reducing CVD. Animal studies demonstrated that CFs are able to decrease cardiac post-ischemic damage via prevention of the mitochondrial permeability transition pore opening, and reduction in superoxide production (104); flavanols might also affect mitochondrial structure and function via stimulation of mitochondrial biogenesis (105). Patients, randomized to receive dark chocolate, showed increased maximal oxygen uptake and maximum work achieved, as well as increases in mitochondrial activity and glutathione levels, when compared to placebo (106). NO is suspected to mediate the effects of cocoa on mitochondria. In support of this thesis, a recent study demonstrated, in vitro, that flavanols are capable to stimulate mitochondrial function and biogenesis; effects disappeared with the inhibition of eNOS $(107,108)$.

Cocoa flavanols are also able to inhibit platelet activation, adhesion, and aggregation, mechanisms that play a central role in the development of endothelial dysfunction and atherosclerosis (109). Indeed, activated platelets secrete a number of adhesion molecules, such as P-selectin and C40 ligand, release inflammatory mediators into the local microenvironment (110), stimulate the chemotaxis of leukocytes to the site of inflammation (111), and generate ROS, reducing NO bioavailability and contributing to endothelial dysfunction and thrombosis (112).

Moreover, cocoa and its main flavanols may improve vascular function by regulating the glucose and lipid profile (113), crucial risk factors for vascular damage. There is evidence that CFs are able to modulate insulin secretion in $\beta$-pancreatic cells, target insulin-sensitive tissues, repress glucose production, enhance glucose uptake through the promotion of glucose transport, and improve lipid metabolism (114).

Altogether, these mechanisms might determine the antihypertensive and cardiovascular protective effects of flavanols in vivo (Figure 1).

\section{CONCLUSION}

Polyphenol-rich foods, such as fruit, vegetables, wine, olive oil, and cocoa, are able to reduce cardiovascular risk and prevent cardiovascular events and death $(3-5,115)$. Among them, cocoa beans have always been of particular interest, as they are one of the richest polyphenol sources. In this context, several epidemiological studies suggest a strong correlation between daily cocoa intake and better cardiovascular outcome in different population settings (19-21). Clinical interventional studies demonstrated a positive effect of flavanol-rich cocoa or chocolate intake on $\mathrm{BP}$ 
reduction and improvement in microvascular and macrovascular function $(116,117)$. In vitro and in vivo studies identified increased NO availability, increased NO synthase activity, and inhibition of ACE as putative mechanisms of this beneficial effect (118).

Cocoa consumption has been demonstrated to improve endothelial function and to lower BP in healthy subjects, in patients with risk factors and hypertension, and in patients with coronary heart disease and heart failure. Furthermore, a 3-mmHg reduction in SBP has been estimated to lower the relative risk of CHD by $5 \%$ and the risk of global mortality by $4 \%$ (119). Thus, the introduction of a moderate amount of flavanol-rich cocoa in the daily diet may be a promising strategy to improve cardiovascular outcomes.

However, commercial chocolate with its high sugar and fat content may be undesirable in a population with increased cardiovascular risk. Furthermore, during the cocoa beans manufacturing process, the total amount of flavanols can be reduced more than 10 -fold by fermentation or roasting (18). This results in an unpredictable content of polyphenols in most commercially available products. Moreover, the optimal dose of daily flavanol intake is still unclear. The EFSA recommends consuming $200 \mathrm{mg}$ of CFs per day, provided by $2.5 \mathrm{~g}$ of high-flavanol cocoa powder

\section{REFERENCES}

1. Oliveira GB, Avezum A, Roever L. Cardiovascular disease burden: evolving knowledge of risk factors in myocardial infarction and stroke through population-based research and perspectives in global prevention. Front Cardiovasc Med (2015) 2:32. doi:10.3389/fcvm.2015.00032

2. Widmer RJ, Flammer AJ, Lerman LO, Lerman A. The Mediterranean diet, its components, and cardiovascular disease. Am J Med (2015) 128(3):229-38. doi:10.1016/j.amjmed.2014.10.014

3. de Lorgeril M, Salen P, Caillat-Vallet E, Hanauer MT, Barthelemy JC, Mamelle N. Control of bias in dietary trial to prevent coronary recurrences: the Lyon Diet Heart Study. Eur J Clin Nutr (1997) 51(2):116-22. doi:10.1038/sj.ejcn.1600374

4. Trichopoulou A, Costacou T, Bamia C, Trichopoulos D. Adherence to a Mediterranean diet and survival in a Greek population. N Engl J Med (2003) 348(26):2599-608. doi:10.1056/NEJMoa025039

5. Estruch R, Ros E, Salas-Salvadó J, Covas MI, Corella D, Arós F, et al. Primary prevention of cardiovascular disease with a Mediterranean diet. $N$ Engl J Med (2013) 368(14):1279-90. doi:10.1056/NEJMoa1200303

6. Tsao R. Chemistry and biochemistry of dietary polyphenols. Nutrients (2010) 2(12):1231-46. doi:10.3390/nu2121231

7. Hooper L, Kroon PA, Rimm EB, Cohn JS, Harvey I, Le Cornu KA, et al. Flavonoids, flavonoid-rich foods, and cardiovascular risk: a meta-analysis of randomized controlled trials. Am J Clin Nutr (2008) 88(1):38-50.

8. Pérez-Jiménez J, Neveu V, Vos F, Scalbert A. Identification of the 100 richest dietary sources of polyphenols: an application of the Phenol-Explorer database. Eur J Clin Nutr (2010) 64(Suppl 3):S112-20. doi:10.1038/ejcn.2010.221

9. Verna R. The history and science of chocolate. Malays J Pathol (2013) 35(2):111-21.

10. Manach C, Scalbert A, Morand C, Rémésy C, Jiménez L. Polyphenols: food sources and bioavailability. Am J Clin Nutr (2004) 79(5):727-47.

11. Arts IC, van De Putte B, Hollman PC. Catechin contents of foods commonly consumed in The Netherlands. 2. Tea, wine, fruit juices, and chocolate milk. J Agric Food Chem (2000) 48(5):1752-7. doi:10.1021/jf000025h

12. McShea A, Ramiro-Puig E, Munro SB, Casadesus G, Castell M, Smith MA. Clinical benefit and preservation of flavonols in dark chocolate manufacturing. Nutr Rev (2008) 66(11):630-41. doi:10.1111/j.1753-4887.2008.00114.x

13. Kothe L, Zimmermann BF, Galensa R. Temperature influences epimerization and composition of flavanol monomers, dimers and trimers during cocoa bean roasting. Food Chem (2013) 141(4):3656-63. doi:10.1016/j. foodchem.2013.06.049

14. Andres-Lacueva C, Monagas M, Khan N, Izquierdo-Pulido M, Urpi-Sarda $\mathrm{M}$, Permanyer J, et al. Flavanol and flavonol contents of cocoa powder or $10 \mathrm{~g}$ of high-flavanol dark chocolate in the context of a balanced diet, but this dose has recently been subject of discussion (80). Thus, a comparison among studies is difficult, because of the heterogeneity between trials, in terms of study population and design, flavanol doses in active and control groups, and study duration.

Further clinical studies are required in order to identify the correct dose and the right modality of manufacturing of flavanolrich cocoa to be able to benefit from daily consumption of this natural medicinal product in the field of CVD.

\section{AUTHOR CONTRIBUTIONS}

All authors made substantial contributions to the conception of this review. VL, JB, MN, and IS drafted the work; FE, CF, AF, and FR revised it critically for important intellectual content. All authors approved the final version of the paper.

\section{FUNDING}

The work was support by Swiss Heart Foundation (AF and IS). products: influence of the manufacturing process. J Agric Food Chem (2008) 56(9):3111-7. doi:10.1021/jf0728754

15. Rein D, Lotito S, Holt RR, Keen CL, Schmitz HH, Fraga CG. Epicatechin in human plasma: in vivo determination and effect of chocolate consumption on plasma oxidation status. J Nutr (2000) 130(8S Suppl):2109S-14S.

16. Serafini M, Bugianesi R, Maiani G, Valtuena S, De Santis S, Crozier A. Plasma antioxidants from chocolate. Nature (2003) 424(6952):1013. doi:10.1038/4241013a

17. Richelle M, Tavazzi I, Enslen M, Offord EA. Plasma kinetics in man of epicatechin from black chocolate. Eur J Clin Nutr (1999) 53(1):22-6. doi:10.1038/ sj.ejcn. 1600673

18. Ellam S, Williamson G. Cocoa and human health. Annu Rev Nutr (2013) 33:105-28. doi:10.1146/annurev-nutr-071811-150642

19. Mink PJ, Scrafford CG, Barraj LM, Harnack L, Hong CP, Nettleton JA, et al. Flavonoid intake and cardiovascular disease mortality: a prospective study in postmenopausal women. Am J Clin Nutr (2007) 85(3):895-909.

20. Buijsse B, Feskens EJ, Kok FJ, Kromhout D. Cocoa intake, blood pressure, and cardiovascular mortality: the Zutphen Elderly Study. Arch Intern Med (2006) 166(4):411-7. doi:10.1001/.411

21. Janszky I, Mukamal KJ, Ljung R, Ahnve S, Ahlbom A, Hallqvist J. Chocolate consumption and mortality following a first acute myocardial infarction: the Stockholm Heart Epidemiology Program. J Intern Med (2009) 266(3):248-57. doi:10.1111/j.1365-2796.2009.02088.x

22. Ried K, Sullivan TR, Fakler P, Frank OR, Stocks NP. Effect of cocoa on blood pressure. Cochrane Database Syst Rev (2012) 8:CD008893. doi:10.1002/14651858.CD008893.pub2

23. Grassi D, Desideri G, Necozione S, di Giosia P, Barnabei R, Allegaert L, et al. Cocoa consumption dose-dependently improves flow-mediated dilation and arterial stiffness decreasing blood pressure in healthy individuals. J Hypertens (2015) 33(2):294-303. doi:10.1097/HJH.0000000000000412

24. Pearson DA, Paglieroni TG, Rein D, Wun T, Schramm DD, Wang JF, et al. The effects of flavanol-rich cocoa and aspirin on ex vivo platelet function. Thromb Res (2002) 106(4-5):191-7. doi:10.1016/S0049-3848(02)00128-7

25. Serafini M, Peluso I. Functional foods for health: the interrelated antioxidant and anti-inflammatory role of fruits, vegetables, herbs, spices and cocoa in humans. Curr Pharm Des (2016) 22(44):6701-15. doi:10.2174/13816128236 66161123094235

26. Mellor DD, Sathyapalan T, Kilpatrick ES, Beckett S, Atkin SL. High-cocoa polyphenol-rich chocolate improves HDL cholesterol in Type 2 diabetes patients. Diabet Med (2010) 27(11):1318-21. doi:10.1111/j.1464-5491.2010.03108.x 
27. Sansone R, Rodriguez-Mateos A, Heuel J, Falk D, Schuler D, Wagstaff R, et al. Cocoa flavanol intake improves endothelial function and Framingham Risk Score in healthy men and women: a randomised, controlled, double-masked trial: the Flaviola Health Study. Br J Nutr (2015) 114(8):1246-55. doi:10.1017/ S0007114515002822

28. Rostami A, Khalili M, Haghighat N, Eghtesadi S, Shidfar F, Heidari I, et al. High-cocoa polyphenol-rich chocolate improves blood pressure in patients with diabetes and hypertension. ARYA Atheroscler (2015) 11(1):21-9.

29. Flammer AJ, Sudano I, Wolfrum M, Thomas R, Enseleit F, Périat D, et al. Cardiovascular effects of flavanol-rich chocolate in patients with heart failure. Eur Heart J (2012) 33(17):2172-80. doi:10.1093/eurheartj/ehr448

30. Paillard F. [Effects of chocolate consumption on physiology and cardiovascular diseases]. Presse Med (2014) 43(7-8):848-51. doi:10.1016/j. lpm.2014.05.005

31. McCullough ML, Chevaux K, Jackson L, Preston M, Martinez G, Schmitz $\mathrm{HH}$, et al. Hypertension, the Kuna, and the epidemiology of flavanols. J Cardiovasc Pharmacol (2006) 47(Suppl 2):S103-9; discussion 119-21. doi:10.1097/00005344-200606001-00003

32. Hollenberg NK, Martinez G, McCullough M, Meinking T, Passan D, Preston M, et al. Aging, acculturation, salt intake, and hypertension in the Kuna of Panama. Hypertension (1997) 29(1 Pt 2):171-6. doi:10.1161/01. HYP.29.1.171

33. Bayard V, Chamorro F, Motta J, Hollenberg NK. Does flavanol intake influence mortality from nitric oxide-dependent processes? Ischemic heart disease, stroke, diabetes mellitus, and cancer in Panama. Int J Med Sci (2007) 4(1):53-8. doi:10.7150/ijms.4.53

34. K Hollenberg N. Vascular action of cocoa flavanols in humans: the roots of the story. J Cardiovasc Pharmacol (2006) 47(Suppl 2):S99-102; discussion S119-21. doi:10.1097/00005344-200606001-00002

35. Mostofsky E, Levitan EB, Wolk A, Mittleman MA. Chocolate intake and incidence of heart failure: a population-based prospective study of middle-aged and elderly women. Circ Heart Fail (2010) 3(5):612-6. doi:10.1161/ CIRCHEARTFAILURE.110.944025

36. Steinhaus DA, Mostofsky E, Levitan EB, Dorans KS, Håkansson N, Wolk A, et al. Chocolate intake and incidence of heart failure: findings from the Cohort of Swedish Men. Am Heart J (2017) 183:18-23. doi:10.1016/j. ahj.2016.10.002

37. Djousse L, Hopkins PN, North KE, Pankow JS, Arnett DK, Ellison RC. Chocolate consumption is inversely associated with prevalent coronary heart disease: the National Heart, Lung, and Blood Institute Family Heart Study. Clin Nutr (2011) 30(2):182-7. doi:10.1016/j.clnu.2010.08.005

38. Kwok CS, Boekholdt SM, Lentjes MA, Loke YK, Luben RN, Yeong JK, et al. Habitual chocolate consumption and risk of cardiovascular disease among healthy men and women. Heart (2015) 101(16):1279-87. doi:10.1136/ heartjnl-2014-307050

39. Writing Group M, Mozaffarian D, Benjamin EJ, Go AS, Arnett DK, Blaha MJ, et al. Heart disease and stroke statistics-2016 update: a report from the American Heart Association. Circulation (2016) 133(4):e38-360. doi:10.1161/CIR.0000000000000350

40. Ettehad D, Emdin CA, Kiran A, Anderson SG, Callender T, Emberson J, et al. Blood pressure lowering for prevention of cardiovascular disease and death: a systematic review and meta-analysis. Lancet (2016) 387(10022):957-67. doi:10.1016/S0140-6736(15)01225-8

41. Fraga CG, Actis-Goretta L, Ottaviani JI, Carrasquedo F, Lotito SB, Lazarus S, et al. Regular consumption of a flavanol-rich chocolate can improve oxidant stress in young soccer players. Clin Dev Immunol (2005) 12(1):11-7. doi:10. 1080/10446670410001722159

42. Grassi D, Lippi C, Necozione S, Desideri G, Ferri C. Short-term administration of dark chocolate is followed by a significant increase in insulin sensitivity and a decrease in blood pressure in healthy persons. Am J Clin Nutr (2005) 81(3):611-4.

43. Crews WD Jr, Harrison DW, Wright JW. A double-blind, placebo-controlled, randomized trial of the effects of dark chocolate and cocoa on variables associated with neuropsychological functioning and cardiovascular health: clinical findings from a sample of healthy, cognitively intact older adults. Am J Clin Nutr (2008) 87(4):872-80.

44. Mastroiacovo D, Kwik-Uribe C, Grassi D, Necozione S, Raffaele A, Pistacchio $\mathrm{L}$, et al. Cocoa flavanol consumption improves cognitive function, blood pressure control, and metabolic profile in elderly subjects: the Cocoa,
Cognition, and Aging (CoCoA) Study - a randomized controlled trial. Am J Clin Nutr (2015) 101(3):538-48. doi:10.3945/ajcn.114.092189

45. Murphy KJ, Chronopoulos AK, Singh I, Francis MA, Moriarty H, Pike MJ, et al. Dietary flavanols and procyanidin oligomers from cocoa (Theobroma cacao) inhibit platelet function. Am J Clin Nutr (2003) 77(6):1466-73.

46. Engler MB, Engler MM, Chen CY, Malloy MJ, Browne A, Chiu EY, et al. Flavonoid-rich dark chocolate improves endothelial function and increases plasma epicatechin concentrations in healthy adults. J Am Coll Nutr (2004) 23(3):197-204. doi:10.1080/07315724.2004.10719361

47. Shiina Y, Funabashi N, Lee K, Murayama T, Nakamura K, Wakatsuki Y, et al. Acute effect of oral flavonoid-rich dark chocolate intake on coronary circulation, as compared with non-flavonoid white chocolate, by transthoracic Doppler echocardiography in healthy adults. Int J Cardiol (2009) 131(3):424-9. doi:10.1016/j.ijcard.2007.07.131

48. Njike VY, Faridi Z, Shuval K, Dutta S, Kay CD, West SG, et al. Effects of sugar-sweetened and sugar-free cocoa on endothelial function in overweight adults. Int J Cardiol (2011) 149(1):83-8. doi:10.1016/j.ijcard.2009.12.010

49. Taubert D, Berkels R, Roesen R, Klaus W. Chocolate and blood pressure in elderly individuals with isolated systolic hypertension. JAMA (2003) 290(8):1029-30. doi:10.1001/jama.290.8.1029

50. Taubert D, Roesen R, Lehmann C, Jung N, Schömig E. Effects of low habitual cocoa intake on blood pressure and bioactive nitric oxide: a randomized controlled trial. JAMA (2007) 298(1):49-60. doi:10.1001/jama.298.1.49

51. Grassi D, Necozione S, Lippi C, Croce G, Valeri L, Pasqualetti P, et al. Cocoa reduces blood pressure and insulin resistance and improves endothelium-dependent vasodilation in hypertensives. Hypertension (2005) 46(2):398-405. doi:10.1161/01.HYP.0000174990.46027.70

52. Muniyappa R, Hall G, Kolodziej TL, Karne RJ, Crandon SK, Quon MJ. Cocoa consumption for $2 \mathrm{wk}$ enhances insulin-mediated vasodilatation without improving blood pressure or insulin resistance in essential hypertension. Am J Clin Nutr (2008) 88(6):1685-96. doi:10.3945/ajcn.2008.26457

53. Davison K, Berry NM, Misan G, Coates AM, Buckley JD, Howe PR. Doserelated effects of flavanol-rich cocoa on blood pressure. J Hum Hypertens (2010) 24(9):568-76. doi:10.1038/jhh.2009.105

54. Grassi D, Desideri G, Necozione S, Lippi C, Casale R, Properzi G, et al. Blood pressure is reduced and insulin sensitivity increased in glucose-intolerant, hypertensive subjects after 15 days of consuming high-polyphenol dark chocolate. J Nutr (2008) 138(9):1671-6.

55. Davison K, Coates AM, Buckley JD, Howe PR. Effect of cocoa flavanols and exercise on cardiometabolic risk factors in overweight and obese subjects. Int J Obes (2008) 32(8):1289-96. doi:10.1038/ijo.2008.66

56. Monagas M, Khan N, Andres-Lacueva C, Casas R, Urpí-Sardà M, Llorach R, et al. Effect of cocoa powder on the modulation of inflammatory biomarkers in patients at high risk of cardiovascular disease. Am J Clin Nutr (2009) 90(5):1144-50. doi:10.3945/ajcn.2009.27716

57. De Palma R, Sotto I, Wood EG, Khan NQ, Butler J, Johnston A, et al. Cocoa flavanols reduce $\mathrm{N}$-terminal pro-B-type natriuretic peptide in patients with chronic heart failure. ESC Heart Fail (2016) 3(2):97-106. doi:10.1002/ehf2.12077

58. Ried K, Sullivan T, Fakler P, Frank OR, Stocks NP. Does chocolate reduce blood pressure? A meta-analysis. BMC Med (2010) 8:39. doi:10.1186/1741-7015-8-39

59. Desch S, Schmidt J, Kobler D, Sonnabend M, Eitel I, Sareban M, et al. Effect of cocoa products on blood pressure: systematic review and meta-analysis. Am J Hypertens (2010) 23(1):97-103. doi:10.1038/ajh.2009.213

60. Ried K, Fakler P, Stocks NP. Effect of cocoa on blood pressure. Cochrane Database Syst Rev (2017) 4:CD008893. doi:10.1002/14651858.CD008893. pub3

61. Flammer AJ, Anderson T, Celermajer DS, Creager MA, Deanfield J, Ganz P, et al. The assessment of endothelial function: from research into clinical practice. Circulation (2012) 126(6):753-67. doi:10.1161/ CIRCULATIONAHA.112.093245

62. Charakida M, Masi S, Lüscher TF, Kastelein JJ, Deanfield JE. Assessment of atherosclerosis: the role of flow-mediated dilatation. Eur Heart J (2010) 31(23):2854-61. doi:10.1093/eurheartj/ehq340

63. Shechter M, Shechter A, Koren-Morag N, Feinberg MS, Hiersch L. Usefulness of brachial artery flow-mediated dilation to predict long-term cardiovascular events in subjects without heart disease. Am J Cardiol (2014) 113(1):162-7. doi:10.1016/j.amjcard.2013.08.051

64. Kullo IJ, Malik AR, Santos S, Ehrsam JE, Turner ST. Association of cardiovascular risk factors with microvascular and conduit artery function in 
hypertensive subjects. Am J Hypertens (2007) 20(7):735-42. doi:10.1016/j. amjhyper.2007.01.015

65. Rodriguez-Mateos A, Hezel M, Aydin H, Kelm M, Lundberg JO, Weitzberg $\mathrm{E}$, et al. Interactions between cocoa flavanols and inorganic nitrate: additive effects on endothelial function at achievable dietary amounts. Free Radic Biol Med (2015) 80:121-8. doi:10.1016/j.freeradbiomed.2014.12.009

66. Schroeter H, Heiss C, Balzer J, Kleinbongard P, Keen CL, Hollenberg NK, et al. (-)-Epicatechin mediates beneficial effects of flavanol-rich cocoa on vascular function in humans. Proc Natl Acad Sci U S A (2006) 103(4):1024-9. doi:10.1073/pnas.0510168103

67. Heiss C, Sansone R, Karimi H, Krabbe M, Schuler D, Rodriguez-Mateos A, et al. Impact of cocoa flavanol intake on age-dependent vascular stiffness in healthy men: a randomized, controlled, double-masked trial. Age (2015) 37(3):9794. doi:10.1007/s11357-015-9794-9

68. Sansone R, Ottaviani JI, Rodriguez-Mateos A, Heinen Y, Noske D, Spencer JP, et al. Methylxanthines enhance the effects of cocoa flavanols on cardiovascular function: randomized, double-masked controlled studies. Am J Clin Nutr (2017) 105(2):352-60. doi:10.3945/ajcn.116.140046

69. Heiss C, Kleinbongard P, Dejam A, Perré S, Schroeter H, Sies H, et al. Acute consumption of flavanol-rich cocoa and the reversal of endothelial dysfunction in smokers. JAm Coll Cardiol (2005) 46(7):1276-83. doi:10.1016/j. jacc.2005.06.055

70. Hermann F, Spieker LE, Ruschitzka F, Sudano I, Hermann M, Binggeli C, et al. Dark chocolate improves endothelial and platelet function. Heart (2006) 92(1):119-20. doi:10.1136/hrt.2005.063362

71. West SG, McIntyre MD, Piotrowski MJ, Poupin N, Miller DL, Preston AG, et al. Effects of dark chocolate and cocoa consumption on endothelial function and arterial stiffness in overweight adults. Br J Nutr (2014) 111(4):653-61. doi:10.1017/S0007114513002912

72. Balzer J, Rassaf T, Heiss C, Kleinbongard P, Lauer T, Merx M, et al. Sustained benefits in vascular function through flavanol-containing cocoa in medicated diabetic patients a double-masked, randomized, controlled trial. J Am Coll Cardiol (2008) 51(22):2141-9. doi:10.1016/j.jacc.2008.01.059

73. Mellor DD, Madden LA, Smith KA, Kilpatrick ES, Atkin SL. High-polyphenol chocolate reduces endothelial dysfunction and oxidative stress during acute transient hyperglycaemia in type 2 diabetes: a pilot randomized controlled trial. Diabet Med (2013) 30(4):478-83. doi:10.1111/dme.12030

74. Heiss C, Dejam A, Kleinbongard P, Schewe T, Sies H, Kelm M. Vascular effects of cocoa rich in flavan-3-ols. JAMA (2003) 290(8):1030-1. doi:10.1001/ jama.290.8.1030

75. Heiss C, Jahn S, Taylor M, Real WM, Angeli FS, Wong ML, et al. Improvement of endothelial function with dietary flavanols is associated with mobilization of circulating angiogenic cells in patients with coronary artery disease. J Am Coll Cardiol (2010) 56(3):218-24. doi:10.1016/j. jacc.2010.03.039

76. Flammer AJ, Hermann F, Sudano I, Spieker L, Hermann M, Cooper $\mathrm{KA}$, et al. Dark chocolate improves coronary vasomotion and reduces platelet reactivity. Circulation (2007) 116(21):2376-82. doi:10.1161/ CIRCULATIONAHA.107.713867

77. Rassaf T, Rammos C, Hendgen-Cotta UB, Heiss C, Kleophas W, Dellanna F, et al. Vasculoprotective effects of dietary cocoa flavanols in patients on hemodialysis: a double-blind, randomized, placebo-controlled trial. Clin J Am Soc Nephrol (2016) 11(1):108-18. doi:10.2215/CJN.05560515

78. Hooper L, Kay C, Abdelhamid A, Kroon PA, Cohn JS, Rimm EB, et al. Effects of chocolate, cocoa, and flavan-3-ols on cardiovascular health: a systematic review and meta-analysis of randomized trials. Am J Clin Nutr (2012) 95(3):740-51. doi:10.3945/ajcn.111.023457

79. Panel EN. Scientific opinion on the substantiation of a health claim related to cocoa flavanols and maintenance of normal endothelium-dependent vasodilation pursuant to Article 13 (5) of Regulation (EC) No 1924/2006. EFSA J (2012) 10:2809. doi:10.2903/j.efsa.2012.2809

80. Vlachojannis J, Erne P, Zimmermann B, Chrubasik-Hausmann S. The impact of cocoa flavanols on cardiovascular health. Phytother Res (2016) 30(10):1641-57. doi:10.1002/ptr.5665

81. Kluknavsky M, Balis P, Puzserova A, Radosinska J, Berenyiova A, Drobna M, et al. (-)-Epicatechin prevents blood pressure increase and reduces locomotor hyperactivity in young spontaneously hypertensive rats. Oxid Med Cell Longev (2016) 2016:6949020. doi:10.1155/2016/6949020
82. Ramirez-Sanchez I, Maya L, Ceballos G, Villarreal F. (-)-Epicatechin activation of endothelial cell endothelial nitric oxide synthase, nitric oxide, and related signaling pathways. Hypertension (2010) 55(6):1398-405. doi:10.1161/HYPERTENSIONAHA.109.147892

83. Fraga CG, Litterio MC, Prince PD, Calabró V, Piotrkowski B, Galleano M. Cocoa flavanols: effects on vascular nitric oxide and blood pressure. J Clin Biochem Nutr (2011) 48(1):63-7. doi:10.3164/jcbn.11-010FR

84. Schnorr O, Brossette T, Momma TY, Kleinbongard P, Keen CL, Schroeter H, et al. Cocoa flavanols lower vascular arginase activity in human endothelial cells in vitro and in erythrocytes in vivo. Arch Biochem Biophys (2008) 476(2):211-5. doi:10.1016/j.abb.2008.02.040

85. Loke WM, Hodgson JM, Proudfoot JM, McKinley AJ, Puddey IB, Croft KD. Pure dietary flavonoids quercetin and (-)-epicatechin augment nitric oxide products and reduce endothelin-1 acutely in healthy men. Am J Clin Nutr (2008) 88(4):1018-25.

86. Calderón-Garcidueñas L, Mora-Tiscareño A, Franco-Lira M, Cross JV, Engle R, Aragón-Flores M, et al. Flavonol-rich dark cocoa significantly decreases plasma endothelin-1 and improves cognition in urban children. Front Pharmacol (2013) 4:104. doi:10.3389/fphar.2013.00104

87. Schini-Kerth VB, Auger C, Etienne-Selloum N, Chataigneau T. Polyphenol-induced endothelium-dependent relaxations role of NO and EDHF. Adv Pharmacol (2010) 60:133-75. doi:10.1016/ B978-0-12-385061-4.00006-4

88. Steffen Y, Schewe T, Sies H. (-)-Epicatechin elevates nitric oxide in endothelial cells via inhibition of NADPH oxidase. Biochem Biophys Res Commun (2007) 359(3):828-33. doi:10.1016/j.bbrc.2007.05.200

89. Suen J, Thomas J, Kranz A, Vun S, Miller M. Effect of flavonoids on oxidative stress and inflammation in adults at risk of cardiovascular disease: a systematic review. Healthcare (Basel) (2016) 4(3):E69. doi:10.3390/healthcare4030069

90. Carnevale R, Loffredo L, Pignatelli P, Nocella C, Bartimoccia S, Di Santo S, et al. Dark chocolate inhibits platelet isoprostanes via NOX2 down-regulation in smokers. J Thromb Haemost (2012) 10(1):125-32. doi:10.1111/j.1538-7836.2011.04558.x

91. Martin MA, Goya L, Ramos S. Potential for preventive effects of cocoa and cocoa polyphenols in cancer. Food Chem Toxicol (2013) 56:336-51. doi:10.1016/j.fct.2013.02.020

92. Rodríguez-Ramiro I, Martín MA, Ramos S, Bravo L, Goya L. Comparative effects of dietary flavanols on antioxidant defences and their response to oxidant-induced stress on Caco2 cells. Eur J Nutr (2011) 50(5):313-22. doi:10.1007/s00394-010-0139-2

93. Scheid L, Reusch A, Stehle P, Ellinger S. Antioxidant effects of cocoa and cocoa products ex vivo and in vivo: is there evidence from controlled intervention studies? Curr Opin Clin Nutr Metab Care (2010) 13(6):737-42. doi:10.1097/MCO.0b013e32833ec45c

94. Schewe T, Steffen Y, Sies H. How do dietary flavanols improve vascular function? A position paper. Arch Biochem Biophys (2008) 476(2):102-6. doi:10.1016/j.abb.2008.03.004

95. Rabadán-Chávez GM, Reyes-Maldonado E, Quevedo-Corona L, PaniaguaCastro N, Escalona-Cardoso G, Jaramillo-Flores ME. The prothrombotic state associated with obesity-induced hypertension is reduced by cocoa and its main flavanols. Food Funct (2016) 7(12):4880-8. doi:10.1039/ C6FO01165A

96. Kim JE, Son JE, Jung SK, Kang NJ, Lee CY, Lee KW, et al. Cocoa polyphenols suppress TNF-alpha-induced vascular endothelial growth factor expression by inhibiting phosphoinositide 3-kinase (PI3K) and mitogen-activated protein kinase kinase-1 (MEK1) activities in mouse epidermal cells. Br J Nutr (2010) 104(7):957-64. doi:10.1017/S0007114510001704

97. Guruvayoorappan C, Kuttan G. (+)-Catechin inhibits tumour angiogenesis and regulates the production of nitric oxide and TNF-alpha in LPS-stimulated macrophages. Innate Immun (2008) 14(3):160-74. doi:10.1177/1753425908093295

98. Selmi C, Mao TK, Keen CL, Schmitz HH, Eric Gershwin M. The anti-inflammatory properties of cocoa flavanols. Journal of cardiovascular pharmacology. (2006) 47(Suppl 2):S163-71; discussion S172-6.

99. Cienfuegos-Jovellanos E, Quiñones Mdel M, Muguerza B, Moulay L, Miguel $\mathrm{M}$, Aleixandre A. Antihypertensive effect of a polyphenol-rich cocoa powder industrially processed to preserve the original flavonoids of the cocoa beans. J Agric Food Chem (2009) 57(14):6156-62. doi:10.1021/jf804045b 
100. Actis-Goretta L, Ottaviani JI, Fraga CG. Inhibition of angiotensin converting enzyme activity by flavanol-rich foods. J Agric Food Chem (2006) 54(1):229-34. doi:10.1021/jf052263o

101. Oboh G, Ademosun AO, Ademiluyi AO, Omojokun OS, Nwanna EE, Longe KO. In vitro studies on the antioxidant property and inhibition of alpha-amylase, alpha-glucosidase, and angiotensin I-converting enzyme by polyphenol-rich extracts from cocoa (Theobroma cacao) bean. Patholog Res Int (2014) 2014:549287. doi:10.1155/2014/549287

102. Persson IA, Persson K, Hagg S, Andersson RG. Effects of cocoa extract and dark chocolate on angiotensin-converting enzyme and nitric oxide in human endothelial cells and healthy volunteers - a nutrigenomics perspective. J Cardiovasc Pharmacol (2011) 57(1):44-50. doi:10.1097/FJC.0b013e3181fe62e3

103. Tang X, Luo YX, Chen HZ, Liu DP. Mitochondria, endothelial cell function, and vascular diseases. Front Physiol (2014) 5:175. doi:10.3389/ fphys.2014.00175

104. González Arbeláez LF, Ciocci Pardo A, Fantinelli JC, Caldiz C, Ríos JL, Schinella GR, et al. Ex vivo treatment with a polyphenol-enriched cocoa extract ameliorates myocardial infarct and postischemic mitochondrial injury in normotensive and hypertensive rats. J Agric Food Chem (2016) 64(25):5180-7. doi:10.1021/acs.jafc.6b01669

105. Nogueira L, Ramirez-Sanchez I, Perkins GA, Murphy A, Taub PR, Ceballos $\mathrm{G}$, et al. (-)-Epicatechin enhances fatigue resistance and oxidative capacity in mouse muscle. JPhysiol (2011) 589(Pt 18):4615-31. doi:10.1113/ jphysiol.2011.209924

106. Taub PR, Ramirez-Sanchez I, Patel M, Higginbotham E, Moreno-Ulloa A, Román-Pintos LM, et al. Beneficial effects of dark chocolate on exercise capacity in sedentary subjects: underlying mechanisms. A double blind, randomized, placebo controlled trial. Food Funct (2016) 7(9):3686-93. doi:10.1039/c6fo00611f

107. Moreno-Ulloa A, Cid A, Rubio-Gayosso I, Ceballos G, Villarreal F, Ramirez-Sanchez I. Effects of (-)-epicatechin and derivatives on nitric oxide mediated induction of mitochondrial proteins. Bioorg Med Chem Lett (2013) 23(15):4441-6. doi:10.1016/j.bmcl.2013.05.079

108. Ramírez-Sánchez I, Rodríguez A, Moreno-Ulloa A, Ceballos G, Villarreal F. (-)-Epicatechin-induced recovery of mitochondria from simulated diabetes: potential role of endothelial nitric oxide synthase. Diab Vasc Dis Res (2016) 13(3):201-10. doi:10.1177/1479164115620982

109. Jumar A, Schmieder RE. Cocoa flavanol cardiovascular effects beyond blood pressure reduction. J Clin Hypertens (2016) 18(4):352-8. doi:10.1111/ jch. 12715

110. Gawaz M, Langer H, May AE. Platelets in inflammation and atherogenesis. J Clin Invest (2005) 115(12):3378-84. doi:10.1172/JCI27196
111. Henn V, Slupsky JR, Gräfe M, Anagnostopoulos I, Förster R, MüllerBerghaus $G$, et al. CD40 ligand on activated platelets triggers an inflammatory reaction of endothelial cells. Nature (1998) 391(6667):591-4. doi: $10.1038 / 35393$

112. Krotz F, Sohn HY, Pohl U. Reactive oxygen species: players in the platelet game. Arterioscler Thromb Vasc Biol (2004) 24(11):1988-96. doi:10.1161/01. ATV.0000145574.90840.7d

113. Lin X, Zhang I, Li A, Manson JE, Sesso HD, Wang L, et al. Cocoa flavanol intake and biomarkers for cardiometabolic health: a systematic review and meta-analysis of randomized controlled trials. J Nutr (2016) 146(11):232533. doi:10.3945/jn.116.237644

114. Martin MA, Goya L, Ramos S. Antidiabetic actions of cocoa flavanols. Mol Nutr Food Res (2016) 60(8):1756-69. doi:10.1002/mnfr.201500961

115. Keys A, Menotti A, Karvonen MJ, Aravanis C, Blackburn H, Buzina $\mathrm{R}$, et al. The diet and 15-year death rate in the seven countries study. Am J Epidemiol (1986) 124(6):903-15. doi:10.1093/oxfordjournals.aje. a114480

116. Sudano I, Flammer AJ, Roas S, Enseleit F, Ruschitzka F, Corti R, et al. Cocoa, blood pressure, and vascular function. Curr Hypertens Rep (2012) 14(4):279-84. doi:10.1007/s11906-012-0281-8

117. Ferri C, Desideri G, Ferri L, Proietti I, Di Agostino S, Martella L, et al. Cocoa, blood pressure, and cardiovascular health. J Agric Food Chem (2015) 63(45):9901-9. doi:10.1021/acs.jafc.5b01064

118. Fraga CG, Oteiza P. Flavanols and vascular health: molecular mechanisms to build evidence-based recommendations. Free Radic Biol Med (2014) 75(Suppl 1):S12. doi:10.1016/j.freeradbiomed.2014.10.859

119. Whelton PK, He J, Appel LJ, Cutler JA, Havas S, Kotchen TA, et al. Primary prevention of hypertension: clinical and public health advisory from The National High Blood Pressure Education Program. JAMA (2002) 288(15):1882-8. doi:10.1001/jama.288.15.1882

Conflict of Interest Statement: The authors declare that the research was conducted in the absence of any commercial or financial relationships that could be construed as a potential conflict of interest.

Copyright (C) 2017 Ludovici, Barthelmes, Nägele, Enseleit, Ferri, Flammer, Ruschitzka and Sudano. This is an open-access article distributed under the terms of the Creative Commons Attribution License (CC BY). The use, distribution or reproduction in other forums is permitted, provided the original author(s) or licensor are credited and that the original publication in this journal is cited, in accordance with accepted academic practice. No use, distribution or reproduction is permitted which does not comply with these terms. 